“ (C) 2016 IEEE. Personal use of this material is permitted. Permission from IEEE must be obtained for all other uses, in any current or future media, including

reprinting/republishing this material for advertising or promotional purposes, creating new collective works, for resale or redistribution to servers or lists, or reuse of any copyrighted component of this work in other works." 


\title{
Optimal Actuator/Sensor Selection Through Dynamic Output Feedback
}

\author{
Ahmadreza Argha*, Steven W. Su*, and Andrey Savkin ${ }^{\dagger}$
}

\begin{abstract}
This paper is devoted to the problem of optimal selection of a subset of available actuators/sensors through a multi-channel $\mathscr{H}_{2}$ dynamic output feedback controller for continuous linear time invariant systems. Incorporating two extra terms for penalizing the number of actuators and sensors into the optimization objective function, we develop an iterative process to identify the favorable row/column-wise sparse DOF gains. Employing the identified structure, we solve the constructed row/column structured multi-channel $\mathscr{H}_{2}$ DOF problem in order to derive a gain that exploits optimum number of sensors/actuators by which the closed-loop stability is maintained and the performance degradation of the closedloop system is restricted. Through an example we demonstrate the remarkable performance and broad applicability of the proposed approach.
\end{abstract}

Index Terms - Simultaneous actuator/sensor selection, multichannel $\mathscr{H}_{2}$ row-column-sparse dynamic output feedback (DOF) problem, linear matrix inequality.

\section{INTRODUCTION}

The number of components (actuators or sensors) in modern control systems can be very large, and hence, it is often not very feasible to manually find a subset of all available components to meet a specific control objective. Hence, the problem of selecting a configuration of actuators (sensors) from the set of all available actuators (sensors), while the control performance remains in an acceptable level compared to the non-sparse performance, is a well-known problem in the literature of control theory; see e.g. [1]-[6]. This problem can equivalently be considered as the design of a row (column) sparse feedback gain for the underling system. This paper aims to develop a unified framework to systematically design a sparse row-wise and columnwise dynamic output feedback (DOF) gain via convex optimization, while satisfying multi-channel $\mathscr{H}_{2}$ performance specifications. One immediate application of this issue will be in the over-actuated (over-sensed) systems [7]. It is known that one method for reconfiguration strategy of fault tolerant control is usually to build an over-actuated (over-sensed) system first and then design a nominal controller using some of the available components. Hence whenever a fault happens in the system, the configuration of the control system is changed by using some of the redundant components in order to attain the nominal control objective [8].

This problem leads to a difficult combinatorial optimization problem. There are a large number of investigations in

\footnotetext{
* A. Argha, S. W. Su are with the Faculty of Engineering and Information Technology, University of Technology, Sydney, PO Box 123, Broadway, NSW 2007, Australia.

\{Ahmadreza.Argha, Steven.Su\}@uts.edu.au

A. Savkin is with the School of Electrical Engineering and Telecommunications, University of New South Wales, Sydney, NSW 2052 Australia.

A. Savkin@unsw.edu.au
}

the literature in this field, but most of them do not consider dynamical systems. The paper [1] provides a convex sensor selection formulation for a problem with linear measurements. In [2] a method is developed in order to identify sparse subsets of sensors while it minimizes the CramerRao bound of a class of nonlinear measurement models. A genetic algorithm-based method is proposed in [3] for the problem of actuator selection. In [4], a convex semidefinite programming (SDP) characterization of the problem formulation is considered by incorporating certain forms of sparsity in the feedback gain.

Most of the sparse control design approaches in the literature have focused on the problem of sparse static output/state feedback design. This paper instead utilizes DOF scheme to control the system using the measured system outputs. Moreover, to the best knowledge of authors, concurrent actuator and sensor selection problem through DOF has not been researched thoroughly. Further, it should be noted that the sparse row-column-wise feedback design using DOF is a totally different problem compared to the case of row (column) sparse static output (state) feedback design, as the sparsification procedure should be applied only to a certain part of the controller.

A well-developed method to identify sparsity patterns of feedback gains in the literature (see e.g. [9] and [10]) is to solve e.g. the $\mathscr{H}_{2}$ (or the $\mathscr{H}_{\infty}$ ) problem, by incorporating a sparsity promoting penalty function to the objective function. Basically, the so-called reweighted $\ell_{1}$ (REL1) norm, which is known as a convex relaxation of the $\ell_{0}$-norm, is usually exploited to make a convex problem rather than the original combinatorial optimization problem. The weights (entries of the weighting matrix) are then updated at each step inversely proportional to the strength of individual entries of feedback gain in the previous step. This scheme successively applies to the applications that the sparsity is required to be achieved at the entry-wise level, i.e. minimizing the number of communication links in distributed control networks exploiting the so-called bilateral communication scheme [11]. However, in the cases that the sparsity is noted at a group (e.g. row or column) level, the strength of groups of variables (entries of feedback gain) should be considered. We consider $\|\cdot\|_{\text {row }-\ell_{0}}\left(\|\cdot\|_{\text {col }-\ell_{0}}\right)$, that counts the number of nonzero rows (columns) of a matrix, as the row (column) sparsity promoting penalty function and then propose its reweighted convex relaxation. This paper then develop an iterative algorithm, using the relaxed row/column sparsity promoting penalty functions, which is able to penalize the number of sensors and actuators employed in the control system simultaneously. 
Notation: $\left[\Phi_{i j}\right]_{r \times r}$ is a (block) matrix with (block) entries $\Phi_{i j}, i=1, \cdots, r, j=1, \cdots, r \cdot \operatorname{diag}\left[\Phi_{i}\right]_{i=1}^{r}$ is a (block) diagonal matrix with (block) entries $\Phi_{i}, i=1, \cdots, r$. Moreover, $\operatorname{col}\left(v_{i}(t)\right)_{i=1}^{r}$ denotes a (block) vector with (block) entries $v_{i}(t), i=1, \cdots, r$. herm $(F)$, where $F$ is a square matrix, stands for $F+F^{*}$.

\section{Problem Statement and Preliminaries}

\section{A. Problem statement}

Consider the following LTI system,

$$
\begin{aligned}
\dot{x}(t) & =A x(t)+B_{2} u(t)+B_{1} w(t) \\
z(t) & =C_{2} x(t)+D_{2} u(t) \\
y(t) & =C x(t),
\end{aligned}
$$

where $x \in \mathbb{R}^{n}, u \in \mathbb{R}^{m}, y \in \mathbb{R}^{p}$ and $z \in \mathbb{R}^{q}$ are the state vector, control input vector, output vector and $\mathscr{H}_{2}$ performance output vector of system, respectively. The matrices in (1) are constant and of appropriate dimensions. It is also assumed that $\left(A, B_{2}\right)$ is stabilizable and $(A, C)$ is detectable. $w(t)$ is the external disturbance of the system. The main goal of this paper is to synthesise dynamic output feedback (DOF) gains, utilizing optimum number of actuators and sensors, for the system in (1), while satisfying multi-channel $\mathscr{H}_{2}$ performance specifications. In doing so, we need primarily consider the multichannel $\mathscr{H}_{2}$ DOF synthesis. In the following of this section, to avoid the conservatism introduced by the so-called quadratic approach for the design of feedback gains with respect to multichannel $\mathscr{H}_{2}$ (or mixed $\mathscr{H}_{2} / \mathscr{H}_{\infty}$ ) performance specifications, we develop a novel LMI method for the multichannel $\mathscr{H}_{2}$ control problems. Before doing so, let us present some definitions which we will make use of later in this paper.

Definition 1: A matrix is said to be structure matrix if its elements are either 0 or 1 . The structure matrix of a block matrix $Y=\left[Y_{i j}\right]_{m \times n}$ with $Y_{i j} \in \mathbb{R}^{r_{i} \times s_{j}}$ is $\mathrm{S}(Y) \triangleq\left[s_{i j}\right]_{m \times n}$ with

$$
s_{i j}= \begin{cases}0 & \text { if } Y_{i j}=0 \\ 1 & \text { otherwise. }\end{cases}
$$

Definition 2: Two matrices $Y_{1}$ and $Y_{2}$ are said to have the same structure if $\mathrm{S}\left(Y_{1}\right)=\mathrm{S}\left(Y_{2}\right)$.

Definition 3: The matrix $Y_{1}$ with $\mathrm{S}\left(Y_{1}\right) \triangleq\left[s_{i j}^{1}\right]_{m \times n}$ is said to be structurally subset of $Y_{2}$ with $\mathrm{S}\left(Y_{2}\right) \triangleq\left[s_{i j}^{2}\right]_{m \times n}$ while $s_{i j}^{2}-s_{i j}^{1} \geq 0$. We denote this as $\mathrm{S}\left(Y_{1}\right) \subseteq \mathrm{S}\left(Y_{2}\right)$.

\section{B. Controller dynamic}

We represent the DOF controller $\mathscr{K}(s)$ in state-space as:

$$
\begin{aligned}
\dot{x}_{c}(t) & =A_{c} x_{c}(t)+B_{c} y(t) \\
u(t) & =C_{c} x_{c}(t)+D_{c} y(t),
\end{aligned}
$$

where $x_{c} \in \mathbb{R}^{n}$ is the state vector of the controller and $A_{c}, B_{c}$, $C_{c}$ and $D_{c}$ are of appropriate dimensions. Let $\left(A_{c l}, B_{c l}, C_{c l}\right)$ denotes realization of $T_{w z}:=C_{c l}\left(s I-A_{c l}\right)^{-1} B_{c l}$ (the closed loop transfer function from $w(t)$ to $z(t))$, where

$$
\begin{aligned}
A_{c l} & :=\left[\begin{array}{cc}
A+B_{2} D_{c} C & B_{2} C_{c} \\
B_{c} C & A_{c}
\end{array}\right], B_{c l}:=\left[\begin{array}{c}
B_{1} \\
0
\end{array}\right], \\
C_{c l} & :=\left[\begin{array}{ll}
C_{2}+D_{2} D_{c} C & D_{2} C_{c}
\end{array}\right] .
\end{aligned}
$$

\section{LMI characterization}

\section{1) $\mathscr{H}_{2}$ LMI characterization:}

Lemma 1: Let $\left(A_{c l}, B_{c l}, C_{c l}\right)$ denotes realization of $T_{w z}$. The following three statements, involving symmetric matrix variables $X, Z$ and the general matrix variable $G$ are equivalent.

i) $A_{c l}$ is stable and $\left\|C_{c l}\left(s I-A_{c l}\right)^{-1} B_{c l}\right\|_{2}^{2}<\gamma$.

ii) $\exists X>0$ and $Z>0$ such that

$$
\begin{aligned}
& {\left[\begin{array}{cc}
A_{c l} X+X A_{c l}^{T} & \star \\
C_{c l} X & -\gamma I
\end{array}\right]<0,} \\
& {\left[\begin{array}{cc}
-Z & \star \\
B_{c l} & -X
\end{array}\right]<0,} \\
& \operatorname{trace}(Z)<1 .
\end{aligned}
$$

iii) $\exists X>0, Z>0$ and $G$ such that

$$
\begin{aligned}
& {\left[\begin{array}{ccc}
-\left(G+G^{T}\right) & \star & \star \\
A_{c l} G+X+G & -2 X & \star \\
C_{c l} G & 0 & -\gamma I
\end{array}\right]<0,} \\
& {\left[\begin{array}{cc}
-Z & \star \\
B_{c l} & -X
\end{array}\right]<0,} \\
& \operatorname{trace}(Z)<1 .
\end{aligned}
$$

Proof: Refer to Appendix.

Remark 1: The advantage of the item iii) over the LMI condition derived in [12] lies within the smaller dimension of the first LMI in iii) than that of in [12].

It should be emphasized that the specific LMI characterization in (4) enables us to utilize different Lyapunov matrices $\left(X_{h}\right)$ for each of the involved LMI constraints in the problem. Moreover, the advantage of the LMI (4) lies within this fact that the product terms between the matrix $A_{c l}$ and the Lyapunov matrices $\left(X_{h}\right)$ is disappeared. More importantly, the control gain does not depend on the Lyapunov matrix, but the instrumental matrix variable $G$. This feature has a significant implication in the design of controllers satisfying multiple objectives such as the multi-channel $\mathscr{H}_{2}$ (or even mixed multi-channel $\mathscr{H}_{2} / \mathscr{H}_{\infty}$ ) specifications. It is also worth mentioning that, usually, the projection lemma is used, in the field of robust control, to eliminate the variable which contains the controller state-space data, and thus, deal only with a set of LMIs which include less number of decision variables. However, as seen, the proposed methodology here utilizes the projection lemma in the opposite direction, that is, introducing an additional matrix variable $G$ to the problem.

2) Multi-channel $\mathscr{H}_{2}$ synthesis using improved LMI characterizations: Our target is primarily to compute a fullorder DOF $\mathscr{K}(s)$ presented in (2) which meets several performance specifications of the form

$$
\begin{aligned}
& \text { minimize }\left\|T_{w_{k} z_{k}}\right\|_{2} \\
& \text { subject to }\left\|T_{w_{1} z_{1}}\right\|_{2}^{2}<\gamma_{1}, \cdots,\left\|T_{w_{k-1} z_{k-1}}\right\|_{2}^{2}<\gamma_{k-1}, \\
&\left\|T_{w_{k+1} z_{k+1}}\right\|_{2}^{2}<\gamma_{i+1}, \cdots,\left\|T_{w_{\mathcal{N}} z_{\mathcal{N}}}\right\|_{2}^{2}<\gamma_{\mathcal{N}},
\end{aligned}
$$

where $\left\|T_{w_{i} z_{i}}\right\|_{2}:=\left\|L_{i} T_{w z} R_{i}\right\|_{2}$, in which $L_{i}$ and $R_{i}$ are selection matrices that specify which channel is involved in the associated constraint, and notice that $\mathscr{N}$ hereafter denotes the 
number of channels. Furthermore, a realization of the closed loop system $T_{w_{i} z_{i}}$ will be obtained by replacing matrices $B_{1}$, $C_{2}$ and $D_{2}$ by $B_{1, i}, C_{2, i}$ and $D_{2, i}, i=1, \cdots, \mathscr{N}$, respectively, in (1). Notice that, in such a case, the closed-loop performance is ensured by constraining (minimizing) the $\mathscr{H}_{2}$ norm of the closed-loop transfer functions associated with the (input/output) signals $w_{i}=R_{i} w$ and $z_{i}=L_{i} z$; see [13], [14]. Letting each channel to be associated with the LMI constraints in (4), (5), and (6), the desired characterization with multi-channel specifications can be obtained by introducing a different Lyapunov variable $X_{h}$ for every channel and exploiting common variable $G$ for all channels. Hence, it is readily verified that using the part iii) of Lemma 1, the LMI characterization for each channel $l$ can be written as:

$$
\begin{aligned}
& {\left[\begin{array}{ccc}
-\left(G+G^{T}\right) & \star & \star \\
A_{c l} G+X_{l}+G & -2 X_{l} & \star \\
C_{c l, l} G & 0 & -\gamma_{l} I
\end{array}\right]<0,} \\
& {\left[\begin{array}{cc}
-Z_{l} & \star \\
B_{c l, l} & -X_{l}
\end{array}\right]<0,} \\
& \operatorname{trace}\left(Z_{l}\right)<1
\end{aligned}
$$

where $X_{l}>0, Z_{l}>0$ and $G$ are LMI variables. Now the multi-objective problem described in (7) can be set as

minimize $\gamma_{k}$

(MCH2)

subject to (8), (9), and (10) for k-th channel,

(8), (9), and (10) for j-th channel, with given $\gamma_{l}, l=1, \cdots, \mathscr{N}, l \neq k$.

\section{Row-Column-Sparse Multi-Channel $\mathscr{H}_{2}$ DOF}

The aim is to design a feedback gain such that it

- ensures the $\mathscr{H}_{2}$ performances with respect to channels $j=1, \cdots, \mathscr{N}, l \neq k$ which means that for a prescribed closed loop $\mathscr{H}_{2}$ performance $\gamma_{l}>0$, we have $\left\|T_{w_{l} z_{l}}\right\|_{2}^{2}<$ $\gamma_{l}$

- minimizes the $\mathscr{H}_{2}$ performance of the $k$-th channel subject to the above constraints and $\mathrm{S}\left(\mathscr{K}_{a}\right) \subseteq \Gamma_{a}$, $\mathrm{S}\left(\mathscr{K}_{s}\right) \subseteq \Gamma_{s}$, where $\Gamma_{a}$ and $\Gamma_{s}$ are given row and column sparse structures, respectively, and $\mathscr{K}_{a} \triangleq\left[C_{c} D_{c}\right], \mathscr{K}_{s} \triangleq$ $\left[\begin{array}{ll}B_{c}^{T} & D_{c}^{T}\end{array}\right]^{T}$.

Remark 2: It is worth mentioning that each nonzero row of $\mathscr{K}_{a}$ corresponds to an actuator used by the DOF controller, and likewise, each nonzero column of $\mathscr{K}_{s}$ corresponds to a sensor employed by the DOF controller.

This problem can be formulated through an optimization program in decision variables $X_{l}>0, Z_{l}>0, l=1, \cdots, \mathscr{N}$, $G, A_{c}, B_{c}, C_{c}, D_{c}$ and $\gamma_{k}>0$ :

$$
\text { minimize } \gamma_{k} \text { subject to }
$$

(8), (9), and (10), for k-th channel,

(8), (9), and (10), for 1-th channel, with given $\gamma_{l}, l=1, \cdots, \mathscr{N}, l \neq k$, $\mathrm{S}\left(\mathscr{K}_{a}\right) \subseteq \Gamma_{a}$ and $\mathrm{S}\left(\mathscr{K}_{s}\right) \subseteq \Gamma_{s}$,

where $\Gamma_{a}$ and $\Gamma_{s}$ are preset row and column sparse structure matrices respectively. A difficulty in (11) is that it involves nonlinear terms. The nonlinearities can fortunately be eliminated by some appropriate change of controller variables. The change of variables here is performed inspired by the one introduced in [12]. We let the instrumental variable $G$ and $V=G^{-1}$ be of the form:

$$
G:=\left[\begin{array}{ll}
G_{1} & G_{3} \\
G_{2} & G_{4}
\end{array}\right], V:=\left[\begin{array}{ll}
V_{1} & V_{3} \\
V_{2} & V_{4}
\end{array}\right],
$$

where $\operatorname{dim} G_{1}=\operatorname{dim} V_{1}=\operatorname{dim} A$. As stated in e.g. [12] and [15], without loss of generality it can be assumed that $G_{2}$ and $V_{2}$ are invertible. Now consider the following invertible matrices:

$$
\mathrm{\top}_{G}:=\left[\begin{array}{ll}
G_{1} & I \\
G_{2} & 0
\end{array}\right], \mathrm{T}_{V}:=\left[\begin{array}{ll}
I & V_{1} \\
0 & V_{2}
\end{array}\right] .
$$

It can readily be deduced that

$$
V \mathrm{\top}_{G}=\mathrm{\top}_{V}, G \mathrm{\top}_{V}=\mathrm{\top}_{G}
$$

Now, performing congruence transformations $\operatorname{diag}\left(\mathrm{T}_{V}, \mathrm{~T}_{V}, I\right), \operatorname{diag}\left(I, \mathrm{~T}_{V}\right), \operatorname{and} \operatorname{diag}\left(I_{\xi} \otimes \mathrm{T}_{V}, I_{\xi} \otimes \mathrm{T}_{V}, I_{\xi} \otimes\right.$ $\mathrm{T}_{V}$ ) in (8) and (9) respectively, along with the following change of variables:

$$
\begin{aligned}
\tilde{A}_{c}:= & V_{1}^{T} A G_{1}+V_{1}^{T} B_{2} D_{c} C G_{1}+V_{2}^{T} B_{c} C G_{1} \\
& +V_{1}^{T} B_{2} C_{c} G_{2}+V_{2}^{T} A_{c} G_{2}, \\
\tilde{B}_{c}:= & V_{1}^{T} B_{2} D_{c}+V_{2}^{T} B_{c} \\
\tilde{C}_{c}:= & D_{c} C G_{1}+C_{c} G_{2}, \\
\tilde{D}_{c}:= & D_{c} \\
\tilde{X}_{l}:= & \mathrm{\top}_{V}^{T} X_{l} \mathrm{\top}_{V} \\
H:= & G_{1}^{T} V_{1}+G_{2}^{T} V_{2},
\end{aligned}
$$

lead to the LMIs in (18) and (19). Therefore, the sparse row/column-wise multi-channel $\mathscr{H}_{2}$ DOF problem can be recast as an optimization program in decision variables $\tilde{X}_{l}=$ $\left[\begin{array}{cc}\tilde{X}_{l, 1} & \tilde{X}_{l, 2} \\ \tilde{X}_{l, 2}^{T} & \tilde{X}_{l, 3}\end{array}\right]>0, Z_{l}>0, l=1, \cdots, \mathscr{N}, V_{1}, G_{1}, H, \tilde{A}_{c}, \tilde{B}_{c}, \tilde{C}_{c}$, $\tilde{D}_{c}$ and $\gamma_{k}>0$ :

$$
\text { minimize } \quad \gamma_{k} \quad \text { subject to }
$$

(18), (19), and (10), for k-th channel,

(18), (19), and (10), for 1-th channel, with given $\gamma_{l}, l=1, \cdots, \mathscr{N}, l \neq k$,

$$
\mathrm{S}\left(\tilde{\mathscr{K}}_{a}\right) \subseteq \Gamma_{a} \text { and } \mathrm{S}\left(\tilde{\mathscr{K}}_{s}\right) \subseteq \Gamma_{s},
$$

where $\tilde{\mathscr{K}_{a}}:=\left[\begin{array}{ll}\tilde{C}_{c} & \tilde{D}_{c}\end{array}\right]$ and $\tilde{\mathscr{K}_{s}}:=\left[\begin{array}{cc}\tilde{B}_{c} & \tilde{D}_{c}\end{array}\right]$.

Remark 3: It is readily derived that

$$
\begin{aligned}
{\left[\begin{array}{cc}
\tilde{A}_{c} & \tilde{B}_{c} \\
\tilde{C}_{c} & \tilde{D}_{c}
\end{array}\right]=\left[\begin{array}{cc}
V_{2}^{T} & V_{1}^{T} B_{2} \\
0 & I
\end{array}\right] } & {\left[\begin{array}{cc}
A_{c} & B_{c} \\
C_{c} & D_{c}
\end{array}\right]\left[\begin{array}{cc}
G_{2} & 0 \\
C G_{1} & I
\end{array}\right] } \\
& +\left[\begin{array}{cc}
V_{1}^{T} A G_{1} & 0 \\
0 & 0
\end{array}\right] .
\end{aligned}
$$

As a result, it can be seen

$$
\tilde{\mathscr{K}}_{a}=\mathscr{K}_{a}\left[\begin{array}{cc}
G_{2} & 0 \\
C G_{1} & I
\end{array}\right] .
$$




$$
\begin{gathered}
{\left[\begin{array}{ccccc}
-\left(G_{1}+G_{1}^{T}\right) & \star & \star & \star & \star \\
-H^{T}-I & -\left(V_{1}+V_{1}^{T}\right) & \star & \star & \star \\
A G_{1}+B_{2} \tilde{C}_{c}+\tilde{X}_{l, 1}+G_{1} & A+B_{2} \tilde{D}_{c} C+\tilde{X}_{l, 2}+I & -2 \tilde{X}_{l, 1} & \star & \star \\
\tilde{A}_{c}+\tilde{X}_{l, 2}^{T}+H^{T} & V_{1}^{T} A+\tilde{B}_{c} C+\tilde{X}_{l, 3}+V_{1}^{T} & -2 \tilde{X}_{l, 2}^{T} & -2 \tilde{X}_{l, 3} & \star \\
C_{2} G_{1}+D_{2} \tilde{C}_{c} & C_{2}+D_{2} \tilde{D}_{c} C & 0 & 0 & -\gamma_{l} I
\end{array}\right]<0} \\
\\
{\left[\begin{array}{cccc}
-Z & \star & \star \\
B_{1} & -\tilde{X}_{l, 1} & \star \\
V_{1}^{T} B_{1} & -\tilde{X}_{l, 2}^{T} & -\tilde{X}_{l, 3}
\end{array}\right]<0}
\end{gathered}
$$

Thus, since post-multiplication retains the row-wise sparsity, it can be stated that $\mathscr{K}_{a}$ is row-sparse if and only if $\tilde{\mathscr{K}}_{a}$ is row-sparse. Moreover, as

$$
\tilde{\mathscr{K}_{s}}=\left[\begin{array}{cc}
V_{2}^{T} & V_{1}^{T} B_{2} \\
0 & I
\end{array}\right] \mathscr{K}_{s},
$$

and since pre-multiplication retains the column-wise sparsity, then $\mathscr{K}_{s}$ is column-sparse if and only if $\tilde{\mathscr{K}}_{s}$ is column sparse. Obtaining a solution from (SMH2), the DOF controller can readily be found from:

1) computing a full rank factorization $G_{2}^{T} V_{2}$ of $H-G_{1}^{T} V_{1}$ and thus invertible $G_{2}$ and $V_{2}$;

2) solving the equations (14)-(17) for controller matrices $A_{c}$, $B_{c}, C_{c}$ and $D_{c}$.

\section{Actuator/Sensor Selection}

While the previous section considers the design of a multichannel $\mathscr{H}_{2}$ DOF with a priori specified set of actuators and sensors, this section explores favorable row and column sparse DOF gains (selection of actuators and sensors). To do so, an optimization framework, in which the sparsity of the feedback gain is directly incorporated into the objective function, is considered here. This problem can be formulated as:

$$
\operatorname{minimize} \quad \gamma_{k}+\eta_{a}\left\|\tilde{\mathscr{K}_{a}}\right\|_{\text {row }-\ell_{0}}+\eta_{s}\left\|\tilde{\mathscr{K}_{s}}\right\|_{\text {col- } \ell_{0}},
$$

subject to the constraints given in (SMH2) except the structural constraint on $\tilde{\mathscr{K}}_{a}$ and $\tilde{\mathscr{K}}_{s}$, where the row- $\ell_{0}$ (col$\ell_{0}$ ) is a quasi-norm that counts the number of non-zero rows (columns) of $\tilde{\mathscr{K}}_{a}\left(\tilde{\mathscr{K}}_{s}\right)$, and $\eta_{a}>0\left(\eta_{s}>0\right)$ is the regularization parameter that implies the emphasis on the row-sparsity (column-sparsity) of $\tilde{\mathscr{K}}_{a}\left(\tilde{\mathscr{K}}_{s}\right)$; i.e. a larger $\eta_{a}$ $\left(\eta_{s}\right)$ will result in a more row-sparse (column-sparse) $\tilde{\mathscr{K}}_{a}$ $\left(\tilde{\mathscr{K}_{s}}\right)$. Clearly the optimization problem $(\mathrm{RC} 0)$ is a combinatorial one and broadly speaking impossible to solve, as an intractable combinatorial search is required to address this problem. In the literature several alternatives such as $\ell_{1}$-norm or weighted $\ell_{1}$-norm are proposed as convex relaxations of the $\ell_{0}$-quasi-norm [16]. Besides, [16] proposes an iterative scheme, called reweighted $\ell_{1}$ (REL1) minimization, that solves a sequence of weighted minimization problems, which the weights are updated according to the previous iteration's solution. The REL1 algorithm has recently been used by a number of researchers (e.g. see [17], [18]) for the design of sparse controllers for the distributed systems. Notice that the variable selection in the aforementioned papers typically amounts to the selection of important individual variables (elements in the feedback gain) rather than the important groups of variables (rows or columns).

Let us now recast the optimization problem $(\mathrm{RC} 0)$ as

$$
\operatorname{minimize} \gamma_{k}+\eta_{a} f\left(\tilde{\mathscr{K}_{a}}\right)+\eta_{s} g\left(\tilde{\mathscr{K}_{s}}\right)
$$

subject to (18), (19), and (10), for k-th channel,

(18), (19), and (10), for 1-th channel, with given $\gamma_{l}, l=1, \cdots, \mathscr{N}, l \neq k$,

where $f(\cdot)(g(\cdot))$ denotes the relaxed row-sparsity (columnsparsity) promoting function for which different choices will be proposed in the following.

\section{A. Row (column) sparsity promoting penalty function}

Let us consider a relaxed row-sparsity promoting function as

$$
f\left(\tilde{\mathscr{K}}_{a}\right)=\sum_{i, j} W_{a, i}\left|\tilde{\mathscr{K}}_{a, i j}\right|,
$$

where $W_{a, i}$ denotes the weight corresponds to the $i$-th row of $\tilde{\mathscr{K}}_{a}$. One may now resort to update the weights inversely proportional to the $\ell_{1}$-norm of its corresponding row in $\tilde{\mathscr{K}}_{a}$ obtained at the previous iteration:

$$
W_{a, i}^{l}=\frac{1}{\sum_{j}\left|\tilde{\mathscr{K}}_{a, i j}^{(l-1)}\right|+\varepsilon},
$$

and form the weighting matrix as $W_{a}=\operatorname{diag}\left[W_{a, i}\right]_{i=1}^{m}$. One can also imagine a variety of possible norms in place of (22), e.g. $\ell_{2}$-norm and $\ell_{\infty}$-norm. Therefore the update rule in (22) can be revised to:

$$
W_{a, i}^{l}=\frac{1}{\sqrt{\sum_{j}\left|\tilde{\mathscr{K}}_{a, i j}^{(l-1)}\right|^{2}}+\varepsilon},
$$

for $\ell_{2}$-norm, and

$$
W_{a, i}^{l}=\frac{1}{\max _{j}\left(\left|\tilde{\mathscr{K}}_{a, i j}^{(l-1)}\right|\right)+\varepsilon},
$$

for $\ell_{\infty}$-norm. Although we have found the one in (22) to perform well in a wide range of experiments, using $\ell_{2}$-norm or $\ell_{\infty}$-norm can sometimes outperform $\ell_{1}$-norm. Notice that these norms only indicate the method that we update the weighting matrix $W_{a}$, while the sparsity term in the objective function is considered as the one in (21). For the sensor 
selection problem, we can similarly replace column- $\ell_{0}$ by a relaxed column-sparsity promoting function as

$$
g\left(\tilde{\mathscr{K}_{s}}\right)=\sum_{i, j}\left|\tilde{\mathscr{K}}_{s, i j}\right| W_{s, j}
$$

while the update rule e.g. in (22) can be converted to:

$$
W_{s, j}^{l}=\frac{1}{\sum_{i}\left|\tilde{\mathscr{K}}_{s, i j}^{(l-1)}\right|+\varepsilon},
$$

and the weighting matrix is similarly formed as $W_{s}=$ $\operatorname{diag}\left[W_{s, j}\right]_{j=1}^{p}$. Algorithm 1 is presented in Appendix to identify a row-sparse $\tilde{\mathscr{K}}_{a}$ and a column-sparse $\tilde{\mathscr{K}}_{s}$. We denote the obtained structure of the minimization problem (SAS), as $\mathrm{S}\left(\tilde{\mathscr{K}}_{a}\right) \triangleq \Gamma_{a}$ and $\mathrm{S}\left(\tilde{\mathscr{K}}_{s}\right) \triangleq \Gamma_{s}$. Eventually, in order to find the multi-channel $\mathscr{H}_{2}$ DOF associated with the achieved $\Gamma_{a}$ and $\Gamma_{s}$, we turn to the minimization problem in (SMH2).

Remark 4: It should be pointed out that if due to some reasons (e.g. high execution cost or faulty situation), some actuators (sensors) are required to be by-passed, one can penalize those actuators (sensors) by a large initial weight (e.g. $1 / \varepsilon$ ) in the proposed iterative algorithm.

\section{NUMERICAL EXAMPLES}

A numerical example is presented here in order to evaluate the effectiveness of the proposed method for the problem of actuator-sensor selection via DOF approach. All the LMI optimization problems are solved by YALMIP [19] as the interface and SDPT3 [20] as the solver.

Let us consider the problem HE3 from COMPleib [21]. This problem is related to the eight order linearized state space model of the dynamics of the Bell201A-1 helicopter that has four inputs and six outputs. The system matrices can be seen in [21]. Besides, we let $C_{1}=\left[\begin{array}{c}C \\ 04 \times 8\end{array}\right], D_{1}=\left[\begin{array}{c}0_{6 \times 4} \\ I_{4}\end{array}\right]$, $C_{2}=\left[\begin{array}{c}\tilde{C} \\ 0_{4 \times 8}\end{array}\right]$ and $D_{2}=\left[\begin{array}{c}0_{2 \times 4} \\ I_{4}\end{array}\right]$, where

$$
\tilde{C}=\left[\begin{array}{llllllll}
1 & 0 & 0 & 0 & 0 & 0 & 0 & 0 \\
0 & 0 & 0 & 1 & 0 & 0 & 0 & 0
\end{array}\right] .
$$

The objective is firstly to identify the minimum number of actuators and sensors by which the closed-loop stability is maintained and the performance degradation of the closedloop system is restricted. Solving the optimization problem in (SMH2), with $\mathscr{N}=2, \gamma_{2}<10, \Gamma_{a}=\mathbb{1}_{m \times(n+p)}, \Gamma_{s}=$ $\mathbb{1}_{(n+m) \times p}$, results in a true $H_{2}$ cost of 0.8117 for channel 1 and 0.4608 for channel 2.

Remark 5: Notice that the value of the $\mathscr{H}_{2}$ cost obtained from (SMH2) is not the true one, due to the conservatism introduced by employing common $G, A_{c}, B_{c}, C_{c}$ and $D_{c}$. Nevertheless, the true value can be computed by solving the following Lyapunov equation

$$
X_{i, t r u e} A_{c l}+A_{c l}^{T} X_{i, t r u e}+C_{c l, i}^{T} C_{c l, i}=0 .
$$

One can then find the $\mathscr{H}_{2}$ cost as $\sqrt{\operatorname{trace}\left(B_{c l, i}^{T} X_{i, t r u e} B_{c l, i}\right)}$. Now we solve the optimization problem in (SAS) by utilizing Algorithm 1 in Appendix with $\beta=0.1, \varepsilon=0.0001$ and exploiting the row-sparsity promoting function (21) and the column-sparsity promoting function (25) in addition to
TABLE I

SUMMARY OF THE OBTAINED RESULTS FOR DIFFERENT VALUES OF $\eta_{s}$ AND $\eta_{a}$

\begin{tabular}{ccccc}
\hline$\eta_{a}$ & $\eta_{s}$ & $\mathscr{H}_{2}$ cost (Ch 1) & Sensors & Actuators \\
\hline 0 & 0 & 0.8117 & All & All \\
1 & 0.5 & 0.9946 & $1 \& 2 \& 3$ & $2 \& 3$ \\
5 & 10 & 0.9946 & $2 \& 3$ & $2 \& 3$ \\
\hline
\end{tabular}

the update rule in (23) and its counterpart for the sensor selection, respectively. As increases $\eta_{a}$ and $\eta_{s}$ the underlying $\mathscr{K}_{a}$ and $\mathscr{K}_{s}$ gradually become row-sparse and column-sparse, respectively. For instance, with $\eta_{a}=1$ and $\eta_{s}=0.5$ the algorithm suggests exploiting only the control inputs 2 and 3 , while the controller does not receive information of sensors 4, 5 and 6, but at the expense of about $23 \% \mathrm{H}_{2}$ performance degradation in channel 1 compared to the non-sparse $\mathscr{K}$.

Moreover, letting $\eta_{a}=5$ and $\eta_{s}=10$, Algorithm 1 proposes exploiting sensors 2 and 3 and actuators 2 and 3 $\left(\left\|\mathscr{K}_{a}\right\|_{\text {row }-\ell_{0}}=2\right.$ and $\left.\left\|\mathscr{K}_{s}\right\|_{\text {col- } \ell_{0}}=2\right)$. About $23 \% H_{2}$ performance degradation happens in channel 1 , in this case. The obtained results for different values of $\eta_{s}$ and $\eta_{a}$ is summarized in Table I.

\section{CONClusions}

This paper develops a framework for addressing the issue of selecting an optimal set of actuators (sensors) for dynamical systems, satisfying several performance constraints. Firstly, an LMI-based framework for the design of multichannel $\mathscr{H}_{2}$ DOF gain has been proposed. Besides, this framework is capable of incorporating additional structural constraints as well as, e.g., regional pole placement constraints on the feedback gain matrix. Then a procedure has been developed which includes two stages; the first stage identifies the desirable row-column-sparsity pattern for necessary parts of dynamic feedback gain via iterative processes and then the second one solves the multi-channel $\mathscr{H}_{2}$ problem, augmented by structural constraints. The simulation results illustrated the effectiveness of our proposed approach.

\section{APPENDIX}

\section{A. Proof of Lemma 1}

The equivalence between the first two statements is a standard $\mathscr{H}_{2}$ state feedback synthesis and can be seen e.g. in [22]. We just show the equivalence between the statements ii) and iii). Exploiting the well-known Schur complement, one can show that the first LMI in iii) can be reformulated as

$$
\left[\begin{array}{cc}
-\left(G+G^{T}\right)+\gamma^{-1}\left(C_{c l} G\right)^{T}\left(C_{c l} G\right) & \star \\
A_{c l} G+X+G & -2 X
\end{array}\right]<0 .
$$

Note that as $G^{T}+G>0, G$ is invertible. By performing congruence transformation $\left[\begin{array}{cc}G^{-T} & 0 \\ 0 & X^{-1}\end{array}\right]$ in the above inequality, we obtain

$$
\left[\begin{array}{cc}
-\left(\tilde{G}+\tilde{G}^{T}\right)+\gamma^{-1} C_{c l}^{T} C_{c l} & \star \\
\tilde{X} A_{c l}+\tilde{X}+\tilde{G} & -2 \tilde{X}
\end{array}\right]<0 .
$$


where $\tilde{G}=G^{-1}$ and $\tilde{X}=X^{-1}$. The above inequality can be written as

$$
\left[\begin{array}{cc}
\gamma^{-1} C_{c l}^{T} C_{c l}+ & \star \\
\tilde{X} A_{c l}+\tilde{X} & -2 \tilde{X}
\end{array}\right]+\operatorname{herm}\left(\left[\begin{array}{c}
-I \\
I
\end{array}\right] \tilde{G}\left[\begin{array}{ll}
I & 0
\end{array}\right]\right)<0 .
$$

According to the well-known Projection lemma, the above inequality holds if and only if the following two projection inequalities with respect to $\tilde{G}$ are satisfied:

$$
\begin{aligned}
& {\left[\begin{array}{l}
I \\
I
\end{array}\right]^{T}\left[\begin{array}{cc}
\gamma^{-1} C_{c l}^{T} C_{c l} & \star \\
\tilde{X} A_{c l}+\tilde{X} & -2 \tilde{X}
\end{array}\right]\left[\begin{array}{l}
I \\
I
\end{array}\right]<0} \\
& {\left[\begin{array}{l}
0 \\
I
\end{array}\right]^{T}\left[\begin{array}{lc}
\gamma^{-1} C_{c l}^{T} C_{c l} & \star \\
\tilde{X} A_{c l}+\tilde{X} & -2 \tilde{X}
\end{array}\right]\left[\begin{array}{l}
0 \\
I
\end{array}\right]<0 .}
\end{aligned}
$$

As seen the inequality (29) indeed becomes the trivial inequality $-\tilde{X}<0$ and (28) is equivalent to

$$
\tilde{X} A_{c l}+A_{c l}^{T} \tilde{X}+\gamma^{-1} C_{c l}^{T} C_{c l}<0
$$

which with pre- and post-multiplying by $X=\tilde{X}^{-1}$ leads to

$$
A_{c l} X+X A_{c l}^{T}+\gamma^{-1} X C_{c l}^{T} C_{c l} X<0
$$

The above inequality can simply be written as item $i i)$, using the Schur complement.

\section{B. Iterative algorithm for identifying row-column-sparsity patterns through DOF}

Define the matrices $R_{a}=\left[\begin{array}{ll}0_{(n+p) \times n} & 1_{(n+p) \times m}\end{array}\right]$ and $R_{S}=$ $\left[\begin{array}{ll}0_{(n+m) \times n} & 1_{(n+m) \times p}\end{array}\right]^{T}$. The optimization problem in (SAS), by letting $f\left(\tilde{\mathscr{K}}_{a}\right)$ and $g\left(\tilde{\mathscr{K}}_{s}\right)$ as (21) and (25) respectively, is equivalent to

$$
\operatorname{minimize} \gamma_{k}+\eta_{a} \operatorname{trace}\left(R_{a} \mathscr{W}\right)+\eta_{s} \operatorname{trace}\left(\mathscr{W} R_{s}\right)
$$

subject to (18), (19), and (10), for k-th channel,

(18), (19), and (10), for 1-th channel,

with given $\gamma_{l}, l \neq k, l=1, \cdots, \mathscr{N}$,

$$
-\mathscr{W} \leq \operatorname{diag}\left(I, W_{a}\right) \cdot \tilde{\mathscr{K}} \cdot \operatorname{diag}\left(I, W_{s}\right) \leq \mathscr{W},
$$

where $W_{a}$ and $W_{s}$ denote the weighting matrices and the last inequality is element-wise with $\mathscr{W} \in \mathbb{R}^{(n+m) \times(n+p)}$ whose entries are nonnegative. Besides, to solve the above optimization problem, the following algorithm is utilized:

Algorithm 1: 1) With given $\varepsilon>0, \beta>0, \eta_{a}>0$ and $\eta_{s}>0$, initialize $W_{a}=I_{m}, W_{s}=I_{p}, l=1$ and $\tilde{\mathscr{K}}^{l}=0$.

2) Solve the minimization problem (30) to obtain $\tilde{\mathscr{K}}^{\star}$ and

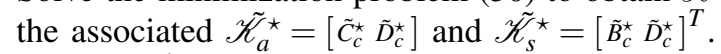

3) Update $W_{a, i}^{l}$ using the update rule in (22) (or (23) or (24)) and its counterpart $W_{s, i}^{l}$ for sensor selection e.g. in (26), form $W_{a}^{l}=\operatorname{diag}\left[W_{a, i}^{l}\right]_{i=1}^{m}$ and $W_{s}^{l}=\operatorname{diag}\left[W_{s, j}^{l}\right]_{j=1}^{p}$.

5) If $\left\|\tilde{\mathscr{K}}^{\star}-\tilde{\mathscr{K}}^{l}\right\| \leq \beta$ go to Step 6 , else $\tilde{\mathscr{K}}^{l}=\tilde{\mathscr{K}}^{\star}, l=$ $l+1$ and return to Step 2 .

6) Let the unnecessary rows of $\tilde{\mathscr{K}}_{a}^{\star}$ and columns of $\tilde{\mathscr{K}}_{s}^{\star}$ be zero and return $\Gamma_{a}^{\star}=\mathrm{S}\left(\tilde{\mathscr{K}}_{a}^{\star}\right)$ and $\Gamma_{s}^{\star}=\mathrm{S}\left(\tilde{\mathscr{K}}_{s}^{\star}\right)$.

\section{REFERENCES}

[1] S. Joshi and S. Boyd, "Sensor selection via convex optimization," Signal Processing, IEEE Transactions on, vol. 57, no. 2, pp. 451-462, 2009.

[2] V. Roy, S. P. Chepuri, and G. Leus, "Sparsity-enforcing sensor selection for doa estimation," in Computational Advances in MultiSensor Adaptive Processing (CAMSAP), 2013 IEEE 5th International Workshop on. IEEE, 2013, pp. 340-343.

[3] J. L. Rogers, "A parallel approach to optimum actuator selection with a genetic algorithm," NASA, Tech. Rep., 2000.

[4] B. Polyak, M. Khlebnikov, and P. Shcherbakov, "An LMI approach to structured sparse feedback design in linear control systems," in Control Conference (ECC), 2013 European. IEEE, 2013, pp. 833-838.

[5] A. V. Savkin, R. J. Evans, and E. Skafidas, "The problem of optimal robust sensor scheduling," Systems \& Control Letters, vol. 43, no. 2 , pp. 149-157, 2001.

[6] A. V. Savkin and R. J. Evans, Hybrid dynamical systems: controller and sensor switching problems. Springer Science \& Business Media, 2002.

[7] A. Cristofaro and T. A. Johansen, "Fault tolerant control allocation using unknown input observers," Automatica, vol. 50, no. 7, pp. 1891$1897,2014$.

[8] T. Steffen, Control reconfiguration of dynamical systems: linear approaches and structural tests. Springer Science \& Business Media, 2005, vol. 320.

[9] F. Lin, M. Fardad, and M. Jovanovic, "Augmented lagrangian approach to design of structured optimal state feedback gains," IEEE Trans. Autom. Control, vol. 56, no. 12, pp. 2923-2929, 2011.

[10] S. Schuler, U. Münz, and F. Allgöwer, "Decentralized state feedback control for interconnected systems with application to power systems," Journal of Process Control, vol. 24, no. 2, pp. 379-388, 2014.

[11] M. Staroswiecki and A. M. Amani, "Fault-tolerant control of distributed systems by information pattern reconfiguration," International Journal of Adaptive Control and Signal Processing, 2014.

[12] P. Apkarian, H. D. Tuan, and J. Bernussou, "Continuous-time analysis, eigenstructure assignment, and $\mathrm{H}_{2}$ synthesis with enhanced linear matrix inequalities (LMI) characterizations," Automatic Control, IEEE Transactions on, vol. 46, no. 12, pp. 1941-1946, 2001.

[13] M. De Oliveira, J. Gerome, and J. Bernussou, "An LMI optimization approach to multiobjective controller design for discrete-time systems," in Decision and Control, 1999. Proceedings of the 38th IEEE Conference on, vol. 4. IEEE, 1999, pp. 3611-3616.

[14] C. Scherer, P. Gahinet, and M. Chilali, "Multiobjective outputfeedback control via LMI optimization," IEEE Transactions on Automatic Control, vol. 42, no. 7, pp. 896-911, 1997.

[15] M. Chilali and P. Gahinet, " $H_{\infty}$ design with pole placement constraints: An LMI approach," Automatic Control, IEEE Transactions on, vol. 41, no. 3, pp. 358-367, 1996.

[16] E. J. Candes, M. B. Wakin, and S. P. Boyd, "Enhancing sparsity by reweighted $\ell_{1}$ minimization," Journal of Fourier analysis and applications, vol. 14, no. 5-6, pp. 877-905, 2008.

[17] M. Fardad and M. R. Jovanovic, "On the design of optimal structured and sparse feedback gains via sequential convex programming," in American Control Conference (ACC), 2014. IEEE, 2014, pp. 24262431.

[18] D. M. Zoltowski, N. Dhingra, F. Lin, and M. R. Jovanovic, "Sparsitypromoting optimal control of spatially-invariant systems," in American Control Conference (ACC), 2014. IEEE, 2014, pp. 1255-1260.

[19] J. Löfberg, "YALMIP: A toolbox for modeling and optimization in MATLAB," in CCA/ISIC/CACSD, Sep. 2004.

[20] K. C. Toh, M. Todd, R. Ttnc, and R. H. Tutuncu, "SDPT3 - a MATLAB software package for semidefinite programming," Optimization Methods and Software, vol. 11, pp. 545-581, 1998.

[21] F. Leibfritz and W. Lipinski, "Description of the benchmark examples in COMPleib 1.0," University of Trier, Tech. Rep., 2003.

[22] S. Boyd, L. E. Ghaoui, E. Feron, and V. Balakrishnan, Linear Matrix Inequalities in System and Control Theory. Philadelphia: Society for Industrial and Applied Mathematics, 1994. 